\section{Bioökonomie als soziotechnische Gestaltungsaufgabe}

\author{
Anforderungen für eine „Good \\ Governance“
}

\begin{abstract}
von Dirk Scheer, Dialogik gemeinnützige Gesellschaft für Kommunikations- und Kooperationsforschung $\mathrm{mbH}$, Stuttgart
\end{abstract}

Wie lässt sich soziotechnischer Wandel im Kontext der Bioökonomie aktiv gestalten? Und wie kann mit Risiko, Unsicherheit, NichtWissen und Komplexität entlang der Wertschöpfungsketten umgegangen werden? Diese Fragestellungen stehen im Mittelpunkt des laufenden Forschungsprojekts ,Good Governance Bioökonomie“" das im Rahmen der sozialwissenschaftlichen Begleitforschung der Bioökonomiestrategie Baden-Württembergs gefördert wird. Im Sinne einer passgenauen Begleitforschung wird das Projekt empirisch auf die bereits geförderten technischen Bioökonomieprojekte im Bereich Lignocellulose und Biogas zurückgreifen, um über Expertengespräche und Interviews Bandbreiten, Einflussfaktoren und Gestaltungspotenziale für den Übergang zu einer biobasierten Wirtschaft zu ermitteln.

\section{Hintergrund}

Das Konzept der Bioökonomie verspricht nicht weniger als die Zusammenführung von Wirtschaft und Nachhaltigkeit. Mit Hilfe wissensbasierter (technologischer) Innovationen wird in zentralen Wirtschaftsbereichen eine Umstellung von fossilen auf erneuerbare, biologische Ressourcen angestrebt, um Produkte, Dienstleistungen und begleitende Prozesse nachhaltig und zugleich zukunfts- und wettbewerbsfähig zu gestalten. Eine biobasierte Wirtschaft soll dazu beitragen, die globalen Herausforderungen zu bewältigen: ausreichende und gesunde Ernährung der Weltbevölkerung, ressourcenschonende und wettbewerbsfähige Rohstoff- und Energieversorgung sowie dauerhafter Umwelt- und Klimaschutz. Mit dem Forschungsprogramm „Bioökonomie Baden-Württemberg" fördert die Landesregierung die bioökonomische Transformation in den drei Bereichen Biogas, Lignocellulose und Mikroalgen. Über eine sozialwissenschaftliche Begleitforschung soll zudem die in der Bioökonomie angelegte Komplexität von Wirkungszusammenhängen erfasst und analysiert werden.

Erfolgreiche Innovationen sind von drei Bedingungen abhängig (Kemp 1997; Voss et al. 2006; Jänicke/Jacob 2006): der Bereitschaft der Anwender, diese Neuerungen einzuführen; der Bereitschaft der späteren Nutzer, die Produkte zu einem wirtschaftlich tragfähigen Preis abzunehmen und der Wirkung von rechtlichen, politischen und ökonomischen Rahmenbedingungen, durch die Chancen verbessert und Risiken verringert werden sollen. Das Projekt „Good Governance Bioökonomie" ist vor allem auf den dritten Einflussbereich der Rahmenbedingungen ausgerichtet und thematisiert (politische) Gestaltungspotenziale eines soziotechnischen Wandels. Kernelemente einer soziotechnischen Steuerungsfähigkeit sind die Charakteristika des Technikfeldes und begleitender Innovationsprozesse, die Konfiguration daran beteiligter Akteure sowie unterschiedliche technikgestaltende Steuerungsansätze (Dierkes/Hoffman 1992; Coenen 2002; Geels 2004; Dolata 2008). Eine Analyse dieser vier Elemente (Technik, Innovation, Akteur, Governance) ist wesentlich (Scheer 2006), um robuste Empfehlungen für eine „Good Governance" der Bioökonomie zu entwickeln.

\section{Das Projekt „Good Governance Bioökonomie"}

\subsection{Projektziele}

Ziel des Forschungsprojekts ist es, die im Rahmen des baden-württembergischen „Forschungsprogramms Bioökonomie“ forcierten Forschungsfelder Biogas und Lignocellulose vergleichend auf die für eine erfolgreiche Umsetzung notwendigen Governance-Strukturen und Implementierungsbedingungen zu untersuchen. Die Konkretisierung erfolgt in beiden Fällen über einen ganzheitlichen Wertschöpfungskettenansatz. Im Bereich Biogasproduktion werden Substratproduktion und -bereitstellung, der Konversionsprozess und die Nutzung der Konversionsprodukte einbezogen. Im Bereich der Lignocellulose stehen Produktion, Konversion und Nutzung von lignocel- 
lulosehaltiger Biomasse für biobasierte chemische Produkte und Energieträger im Vordergrund. Für beide Fallstudien werden die bestehenden und sich abzeichnenden Risiken und Unsicherheiten sowie Komplexitäten und Ambivalenzen entlang der Wertschöpfungskette systematisch und vergleichend untersucht. Neben der systematischen Analyse von Diffusions- und Implementationsbedingungen steht auch die Erarbeitung von Handlungsempfehlungen im Vordergrund, wie die Chancen durch die Setzung von Rahmenbedingungen verbessert und mögliche Risiken bei der Implementierung bioökonomischer Wertschöpfungsketten vermindert werden können.

\subsection{Vorgehensweise}

In einem ersten Schritt werden die soziotechnischen Innovationen von Biogas- und Lignocellulose-Wertschöpfungsketten rekonstruiert und abgebildet. Die Charakterisierung der Wertschöpfungsketten erfolgt über eine detaillierte Beschreibung der Risiken und Unsicherheiten sowie der Komplexität und Ambivalenz entlang der Wertschöpfungsketten. Dieser problemorientierte Zugang dient dazu, die Heterogenität der Bioökonomie aufzuzeigen und unterschiedliche Implikationen auf Politik-, Wirtschafts- und Gesellschaftsbereiche sichtbar zu machen. Auf Basis eines vergleichenden Analyserahmens lassen sich dann Gemeinsamkeiten und Unterschiede entlang der Biogas- und lignocellusosebasierten Produktwege abgleichen.

Anhand dieser vergleichenden Darstellung werden exemplarische Innovationen aus dem Bereich Biogas und Lignocellulose ausgewählt, für die über vertiefende Analysen der Wertschöpfungskette und der dort zu erwartenden Wirkungen Governance-Anforderungen abgeleitet werden. Dafür in Frage kommende Innovationen sind bei der Lignocellulose bspw. der Einsatz von Miscanthus (eine Grasart mit hohem Energiepotenzial) oder Kurzumtriebsplantagen (Anpflanzung schnell wachsender Bäume wie Pappeln oder Weiden), Aufschluss- und Konversionsverfahren wie Pyrolyse oder hydrothermale Verfahren zur Erzeugung von energiereichen, festen oder flüssigen Zwischenprodukten, die dann effizient weiter in Synthesegas umgewandelt werden können.
Auf Produktebene sind Innovationen wie Monomere (insbesondere Zucker-Monomere zur Herstellung chemischer Grundstoffe wie Milchsäure, Essigsäure oder Ethanol), „grüne“ Tenside (aus nachwachsenden Rohstoffen hergestellte Waschund Reinigungsmittel) oder Bionische Composite (z. B. Kunststoffe, Faserverbundwerkstoffe) relevant. Im Anwendungsfeld Biogas beziehen sich Innovationen bspw. auf Biogassubstrate, die überwiegend nicht in Konkurrenz zu Nahrungsmitteln stehen. Dies sind etwa pflanzliche Reststoffe wie Getreideausputz, Melasse, Rapspresskuchen oder auch Nicht-Nahrungspflanzen wie Sudangras oder Zuckerhirse (Weiland 2009). Auch werden Innovationen für die verbesserte Integration von Biogas in das Erdgasnetz in der Analyse mit berücksichtigt. In einem weiteren Schritt werden die vorliegenden Analysen entlang der Wertschöpfungsketten hinsichtlich ihrer Akteurs- und Governance-Struktur analysiert. Daneben wird die Frage behandelt, welche bestehenden Regelungen und rechtlichen Bedingungen bei der Diffusion neuer bioökonomischer Verfahren und Anwendungen gelten.

Darauf aufbauend werden Handlungsempfehlungen für eine aktive Steuerung der Bioökonomie abgeleitet und entwickelt. Dabei werden zunächst unterschiedliche Steuerungskonzepte differenziert, denen verschiedene (politik-)instrumentelle, kommunikative und partizipative Elemente zugrunde liegen. Diese Steuerungskonzepte werden dann von Expertinnen und Experten diskutiert und bewertet. Dies erfolgt über ein Gruppen-Delphi, bei dem die verschiedenen Instrumente und Steuerungsformen von einer interdisziplinär zusammengesetzten Expertenrunde nach den Kriterien der Effektivität, Effizienz, Fairness, Resilienz und Akzeptanz bewertet werden.

Die Delphi-Methode zielt darauf ab, eine möglichst große Bandbreite von Expertenurteilen zu erhalten (Turoff 1970; Pill 1971; Linstone/ Turoff 2002). Die Variante des Gruppen-Delphi orientiert sich im Gegensatz zum traditionellen Delphi an einem Workshop-Konzept (Webler et al. 1991). Inhaltlich geht es bei dem GruppenDelphi um die Entwicklung und Übersetzung bioökonomischer Innovationen und Wertschöpfungsketten in einen sektorübergreifenden Transformationsprozess, bei dem gesellschaftliche, 
technische und marktliche Bedingungen für die erfolgreiche Diffusion angesprochen werden. Dies kann sich bspw. auf folgende übergreifenden Aspekte beziehen:

- notwendige Veränderungen im Nutzer- und Verbraucherverhalten,

- Akzeptanzprobleme und Technikkonflikte um Anbauflächen oder Produktionszyklen,

- Anreizsysteme und geeignete politische und ökonomische Randbedingungen,

- Umgang mit möglicherweise neu auftretenden Risikotypen,

- die Verbindung formeller Planungsverfahren mit stärkerer gesellschaftlicher Partizipation sowie

- adäquate Betreibermodelle für die stoffliche und energetische Nutzung von bioökonomischen Ressourcen.

\section{Fazit}

Die Bioökonomie kommt derzeit vor allem als ein (technologisches) Versprechen mit großer Reichweite daher. Inwieweit dieses Versprechen einzulösen ist, hängt entscheidend von der soziotechnischen Konfiguration und Einbettung der Bioökonomie ab. Die Analyse des Zusammenspiels von Technik, Innovation, Akteur und Governance steht im Mittelpunkt des vorgestellten Projekts „Good Governance Bioökonomie“, bei dem aktive Gestaltungsoptionen des soziotechnischen Wandels im Kontext der Bioökonomie sondiert und Aspekte des Risikos, der Unsicherheit und Komplexität sowie Ambiguität entlang konkreter biobasierter Wertschöpfungsketten untersucht werden.

\section{Literatur}

Coenen, $R$., 2002: Umlenken auf nachhaltige Technologiepfade. In: Grunwald, A. (Hg.): Technikgestaltung für eine nachhaltige Entwicklung. Von der Konzeption zur Umsetzung. Berlin, S. 389-405

Dierkes, M.; Hoffmann, U. (Hg.), 1992: New Technology at the Outset. Social Forces in the Shaping of Technological Innovations. Frankfurt a. M.

Dolata, U., 2008: Soziotechnischer Wandel, Nachhaltigkeit und politische Gestaltungsfähigkeit. In: Lange, H. (Hg.): Nachhaltigkeit als radikaler Wandel. Die Quadratur des Kreises? Wiesbaden, S. 261-286
Geels, F.W., 2004: From Sectoral Systems of Innovation to Socio-technical Systems. Insights About Dynamics and Change from Sociology and Institutional Theory. In: Research Policy 33/6-7 (2004), S. 897-920 Jänicke, M.; Jacob, K. (Hg.), 2006: Environmental Governance in Global Perspective. New Approaches to Ecological Modernisation. FFU-Report 01-2006, Forschungsstelle für Umweltpolitik, FU Berlin

Kemp, R., 1997: Environmental Policy and Technical Change. A Comparison of the Technological Impact of Policy Instruments. Cheltenham

Linstone, H.A.; Turoff, M. (Hg.), 2002: The Delphi Method: Techniques and Applications. New Jersey

Pill, J., 1971: The Delphi Method: Substance, Context, a Critique and an Annotated Bibliography. In: Socio-Economic Planning Sciences 5/1 (1971), S. 57-71

Scheer, D., 2006: Environmental Governance and Integrated Product Policy. In: Scheer, D.; Rubik, F. (Hg.): Governance of Integrated Product Policy. Sheffield, S. 44-68

Turoff, M., 1970: The Design of a Policy Delphi. In: Technological Forecasting and Social Change 2/2 (1970), S. 149-171

Voss, J.-P.; Bauknecht, D.; Kemp, R., (Hg.), 2006: Reflexive Governance for Sustainable Development. Cheltenham

Webler, Th.; Levine, D.; Rakel, H. et al., 1991: A Novel Approach to Reducing Uncertainty: The Group Delphi. In: Technological Forecasting and Social Change 39/3 (1991), S. 253-263

Weiland, P., 2009: Verbesserung der Effizienz und Umweltverträglichkeit von Biogasanlagen. In: Bley, T. (Hg.): Biotechnologische Energieumwandlung: Gegenwärtige Situation, Chancen und künftiger Forschungsbedarf. Berlin, S. 61-71

\section{Kontakt}

Dr. Dirk Scheer

Dialogik gemeinnützige Gesellschaft für Kommuni-

kation- und Kooperationsforschung $\mathrm{mbH}$

Lerchenstraße 22, 70176 Stuttgart

Tel: +49 62216733986

E-Mail: scheer@dialogik-expert.de

\section{$\ll 》$}

